\title{
Erratum zu: V7: Bedarf an hochschulisch qualifizierter Pflege - Präsentation der Ergebnisse einer qualitativen Studie
}

\author{
Anke Simon · Bettina Flaiz $\cdot$ Melanie Elze
}

Online publiziert: 18. Januar 2014

(C) Springer-Verlag Wien 2014

Erratum zu: HeilberufeSCIENCE (2013) 4:26

DOI 10.1007/s16024-013-0187-7

Die Stichprobe besteht aus 13 Experten $(n=13)$, fälschlicherweise steht im Text $(n=12)$.

The Onlineversion des Originalbeitrages ist erreichbar unter doi:http://dx.doi.org/10.1007/s16024-013-0187-7

B. Flaiz $(\bowtie) \cdot$ A. Simon $\cdot$ M. Elze

Duale Hochschule Baden-Württemberg,

Stuttgart, Deutschland

E-Mail: Flaiz@dhbw-stuttgart.de

Springer 